\title{
Heteroskedasticity testing through a comparison of Wald statistics
}

\author{
José M. R. Murteira • Esmeralda A. Ramalho • \\ Joaquim J. S. Ramalho
}

Received: 2 January 2012 / Accepted: 4 March 2013 / Published online: 14 April 2013 (C) ISEG 2013

\begin{abstract}
This paper shows that a test for heteroskedasticity within the context of classical linear regression can be based on the difference between Wald statistics in heteroskedasticity-robust and nonrobust forms. The test is asymptotically distributed under the null hypothesis of homoskedasticity as chi-squared with one degree of freedom. The power of the test is sensitive to the choice of parametric restriction used by the Wald statistics, so the supremum of a range of individual test statistics is proposed. Two versions of a supremum-based test are considered: the first version does not have a known asymptotic null distribution, so the bootstrap is employed to approximate its empirical distribution. The second version has a known asymptotic distribution and, in some cases, is asymptotically pivotal under the null. A simulation study illustrates the use and finite-sample performance of both versions of the test. In this study, the bootstrap is found to provide better size control than asymptotic critical values, namely with heavy-tailed, asymmetric distributions of the covariates. In addition, the use of well-known modifications of the heteroskedasticity consistent covariance matrix estimator of OLS coefficients is also found to benefit the tests' overall behaviour.
\end{abstract}

Keywords Heteroskedasticity testing - White test · Wald test · Supremum

\section{JEL Classification C12}

\footnotetext{
J. M. R. Murteira ( $\square)$

Faculdade de Economia, Universidade de Coimbra and CEMAPRE-ISEG/UTL, Av. Dias da Silva, 165, 3004-512, Coimbra, Portugal

e-mail: jmurt@fe.uc.pt

E. A. Ramalho · J. J. S. Ramalho

Departamento de Economia, CEFAGE-UE, Universidade de Évora,

Largo dos Colegiais 2, 7000-803, Évora, Portugal
} 